\title{
FACTORES DE RIESGO ASOCIADOS A LA TRANSMISIÓN VERTICAL DEL VIRUS DE INMUNODEFICIENCIA HUMANA DURANTE LA GESTACIÓN
}

\section{RISK FACTORS ASSOCIATED WITH VERTICAL TRANSMISSION OF THE HUMAN IMMUNODEFICIENCY VIRUS DURING GESTATION}

\author{
Katherin Faviola Moreno Reyes ${ }^{1, a}$, Félix Dasio Ayala Peralta1,2,b ${ }^{1,2}$ Carlos Velásquez Vásquez ${ }^{1, c}$
}

\begin{abstract}
RESUMEN
La infección por el virus de la inmunodeficiencia humana $(\mathrm{VIH})$ continúa siendo un problema de salud perinatal a nivel global y en nuestro país, por el incremento de contagio en mujeres embarazadas que corren el riesgo para la transmisión vertical del VIH hacia su bebé durante el embarazo, parto y lactancia materna.

Diversas publicaciones mencionan que la prevalencia de transmisión vertical del VIH varía según distintos autores entre $3,8 \%$ a $17 \%$. En el Perú se reporta en $4 \%$.

Entre los factores de riesgo asociados a transmisión vertical del VIH se mencionan: ausencia de terapia antirretroviral durante la atención prenatal con razón de posibilidades ajustada (ORa) que varían entre 2,41 a 17,20; ausencia de programa de prevención de la transmisión de madre a hijo del VIH con ORa de 4,6 a 40,6; ausencia de control prenatal ORa 4,6; parto domiciliario con ORa de 3,35 a 8,10; parto por cesárea de emergencia con OR de 4,32; ausencia de profilaxis antirretroviral al recién nacido ORa de 3,4 a 5,83 y alta carga viral en el embarazo entre $4,0 \%$ a 8,5\%.
\end{abstract}

Palabras claves: Factores de riesgo; Transmisión vertical del VIH; Virus de inmunodeficiencia humana; Infección por VIH durante la gestación (fuente DeCS BIREME).

\begin{abstract}
Infection by the human immunodeficiency virus (HIV) continues to be a perinatal health problem globally and in our country, due to the increase in contagion in pregnant women who run the risk of vertical transmission of HIV to their baby during the pregnancy, childbirth and breastfeeding.

Various publications mention that the prevalence of vertical transmission of HIV varies according to different authors between $3.8 \%$ and $17 \%$. In Peru it is reported at $4 \%$.

Among the risk factors associated with vertical transmission of HIV, the following are mentioned: absence of antiretroviral therapy during prenatal care with adjusted odds ratio (aOR) ranging from 2.41 to 17.20; absence of a program for the prevention of mother-to-child transmission of HIV with aORa of 4.6 to 40.6; absence of prenatal control ORa 4.6; home delivery with aOR of 3.35 to 8.10 ; emergency cesarean delivery with OR of 4.32; absence of antiretroviral prophylaxis to the newborn ORa of 3.4 to 5.83 and high viral load in pregnancy between $4.0 \%$ to $8.5 \%$.
\end{abstract}

Keywords: Risk factors; Vertical transmission of HIV; Human immunodeficiency virus; HIV infection during pregnancy (Source MeSH NLM).

\section{INTRODUCCIÓN}

La infección por el virus de la inmunodeficiencia humana $(\mathrm{VIH})$ continúa siendo un problema de salud perinatal a nivel global y en el país, sobre todo en gestantes infectadas con VIH, que corren el riesgo para la transmisión vertical del VIH hacia su bebé durante el embarazo, parto y lactancia materna.
Según la Organización Mundial de la Salud, cada año, alrededor de 1,4 millones de mujeres infectadas con VIH quedan embarazadas en el mundo. Si no reciben tratamiento antirretroviral, tienen entre $15 \%$ y $45 \%$ de posibilidades de transmitir el virus a sus hijos, pero con intervenciones eficaces permiten reducir esas cifras a niveles inferiores al $5 \%$ 1,2.

\footnotetext{
Instituto Nacional Materno Perinatal. Lima-Perú.

Universidad Nacional Mayor de San Marcos. Lima-Perú.

a Licenciada en Obstetricia. Asistente de Unidad de Referencias y Contrarreferencias.

Médico GínecoObstetra. Profesor Ordinario del Departamento de Ginecología y Obstetricia.

iD Código ORCID: https://orcid.org/0000-0002-2830-3789, Félix Dasio Ayala Peralta

Médico Pediatra. Asistente del Departamento de Neonatología. Experto en ITS-VIH/SIDA

Citar como: Moreno Reyes KF, Ayala Peralta FD, Velasquez-Vasquez C. Factores de riesgo asociados a la transmisión vertical del virus de inmunodeficiencia humana durante la gestacion. Rev Peru Investig Matern Perinat 2021; 10(1): 27-36 DOI https://doi.org/10.33421/inmp.2021229
}

Recibido: 22-12-2020 Aprobado: 31-03-2021 
Diversas publicaciones internacionales mencionan que la prevalencia de transmisión vertical del VIH varía según distintos autores entre $3,8 \%$ a $17 \%$. En el Perú recientes estudios de Velasquez-Vasquez $\mathrm{C}$ et $\mathrm{al}^{3}$ reportan la incidencia de transmisión materno infantil en aproximadamente $4 \%$; los cuales están muy alejados de la meta propuesta por la OMS/OPS que es menor del $2 \% 4$.

La trasmisión vertical, también llamada trasmisión perinatal o transmisión materno infantil, es el proceso infeccioso que implica el paso del $\mathrm{VIH}$ de una mujer embarazada infectada a su hijo durante la gestación, el trabajo de parto y durante la lactancia materna ${ }^{5-8}$.

La transmisión vertical se puede producir durante el embarazo en $25-40 \%$ de casos, durante el trabajo de parto en $60-75 \%$ de casos y por lactancia materna en $16 \%$ de $\operatorname{casos}^{8,9}$.

Los principales factores asociados que incrementan el riesgo de transmisión de la infección por el VIH de madre a hijo incluyen: aumento de la carga viral en la gestante, falta de terapia antirretroviral (TAR) o interrupción de TAR durante el embarazo y la lactancia, infección aguda por VIH durante el embarazo o la lactancia, lactancia materna y lactancia mixta, infecciones del tracto genital, secreciones cervicovaginales, trabajo de parto prolongado, ruptura de membranas amnióticas, embarazos múltiples, monitoreo invasivo y partos instrumentados ${ }^{1,2,10}$.

Cabe precisar que, para la transmisión vertical hacia el feto, intervienen múltiples factores de riesgo, entre los que se mencionan: grado de estadio de la infección de VIH durante la gestación, su carga viral, recuento de linfocitos CD4, infección reciente durante la gestación, infecciones concomitantes, realización de técnicas invasivas y administración de terapia antirretroviral durante la gestación ${ }^{2,11}$.

Asimismo, existen factores de riesgo obstétricos como son el tipo de parto vía vaginal o vía cesárea que influirían en la transmisión del $\mathrm{VIH}$; si la gestante tiene ruptura prematura de membranas aumentaría el riesgo de contagio perinatal; así como, la lactancia materna es considerada como factor de riesgo peligroso para la transmisión del VIH hacia el recién nacido. De todos estos factores el estado de salud de la madre es posiblemente el factor que más influye en el riesgo de transmisión del $\mathrm{VIH}^{2,11}$.

Por otro lado, juega un rol importante para la infección por el VIH, el tipo de exposición al VIH; y ésta puede ser adquirida a través de: transfusión sanguínea, transmisión por contacto sexual, y de gestantes infectadas que pueden transmitir a su bebé durante el embarazo, parto y lactancia materna. De todas ellas, la forma más común de contagio del VIH es a través del contacto sexual con una pareja infectada 2,11
Dentro de los factores epidemiológicos que más influyen en el riesgo de transmisión del VIH están el estado de salud general de la gestante, el grado de infección que posee incluida su estado de inmunodeficiencia severa o si su carga viral está muy elevada. Asimismo, el consumo de drogas durante el embarazo; el acceso al control prenatal; el tratamiento antirretroviral que reciban o la existencia de otras enfermedades coadyuvantes durante el embarazo. Las mujeres muy desnutridas también tienen más riesgo de transmitir la infección con $\mathrm{VIH}^{2,11}$.

Asimismo, señalar que, entre los factores asociados a las características de la población, se mencionan también patrones de comportamiento sexual como la alta prevalencia de relaciones sexuales extramaritales, una cultura sexual más permisiva, iniciación temprana de la actividad sexual y la proliferación de poliandria, homosexualidad, entre otras, que contribuyen a que la infección del VIH se propague, afectando principalmente a la gestante y poniendo el riesgo fetal y perinatal ${ }^{12-15}$.

En el Perú, hay pocos reportes relacionados a transmisión vertical; según Alarcón $\mathrm{J}$ y colaboradores ${ }^{16}$ la transmisión vertical es una de las principales formas de adquisición del VIH en el niño ; así como, García $\mathrm{PJ}$ et $a{ }^{17}$ identifican algunos determinantes para la infección del VIH en la gestante, señalando que los riesgos se encontraban en el momento del embarazo y en las barreras para el acceso oportuno a los establecimientos de salud. Asimismo, Velásquez $\mathrm{C}^{18}$ reporta que al comparar los protocolos de prevención de la transmisión materno infantil de VIH aplicados en el Perú y su efecto en las pacientes atendidas en el Instituto Nacional Materno Perinatal, encontró una disminución de la transmisión materno infantil desde un $15 \%$, cuando se utilizaba solo zidovudina como profilaxis en la gestante con $\mathrm{VIH}$, hasta un $4 \%$, cuando comenzó a utilizarse el tratamiento de gran actividad o TARGA. Por su parte, Huamán $\mathrm{B}$ et $\mathrm{al}^{19}$ identificaron factores que se constituyen en barreras para la aplicación de los protocolos de la prevención de transmisión vertical del VIH en poblaciones amazónicas. Asimismo, Grández-Urbina JA et $\mathrm{al}^{20}$ evidencia la necesidad de un urgente enfoque médico antropológico para detener la transmisión de $\mathrm{VIH}$ en las comunidades indígenas de la amazonia.

Estudio reciente de Velasquez-Vasquez C y $\mathrm{col}^{3}$ mostraron que la mayoría de los niños con VIH por transmisión materno infantil proceden de madres que no recibieron tratamiento antirretroviral durante la gestación, la mayoría de ellas no cuenta con registro de pruebas de $\mathrm{VIH}$ realizada durante la gestación o presentó un resultado negativo; el mayor porcentaje de niños con VIH por transmisión vertical presentan un diagnóstico tardío, gozan por lo general de un buen estado de salud, mientras que en menor porcentaje se encontró desnutrición. 
Cabe precisar que uno de los factores de mucha importancia en una mujer embarazada infectada con VIH es la atención prenatal con el propósito de recibir tratamiento antirretroviral altamente efectiva a partir de 13-16 semanas de gestación para conseguir una carga viral suprimida o indetectable ${ }^{7}$; ya que la base del tratamiento para conseguir que la embarazada alcance la supresión de la carga viral (menos de mil copias por mililitro) o que ésta sea indetectable (menos de 50 copias por $\mathrm{ml}$ ) es la terapia antirretroviral ${ }^{7,21}$. Al contrario, un cuidado prenatal inadecuado, es una importante razón para la falta de conocimiento de las madres sobre la prevención de la transmisión vertical del HIV, lo que puede impedir el uso de los servicios de salud².

Por ello, diversas publicaciones internacionales consideran que las gestantes infectadas con VIH deben recibir tratamiento antirretroviral independientemente de su nivel de linfocitos CD4 con la finalidad de prevenir la transmisión vertical durante la gestación ${ }^{22}$.

Asimismo, tal como indica la Norma técnica de salud para la prevención de la transmisión materno infantil del VIH en el Perú ${ }^{8}$ la mujer que acude a la consulta con embarazo temprano y desconoce su estado serológico debe indicársele una prueba rápida para detección del VIH en la primera consulta prenatal.

La estrategia de manejo en la gestante infectada con $\mathrm{VIH}$ debe efectuarse durante la atención prenatal, en el intraparto, y durante la lactancia materna con la identificación de factores epidemiológicos y obstétricos: uso de la terapia antirretroviral en forma precoz en el primer trimestre de la gestación; ya que la eficacia de la prevención de la transmisión perinatal del VIH depende tanto de la carga viral, como el inicio precoz y la duración de terapia antirretroviral durante la gestación ${ }^{6}$.

Por otro lado, el Colegio Americano de Obstetricia y Ginecología (ACOG) ${ }^{2}$ menciona que el riesgo de transmisión de madre a hijo en gestantes infectadas por el VIH con cargas virales elevadas puede reducirse realizando cesárea antes del inicio de trabajo de parto y antes de la rotura de membranas, de preferencia cesárea programada a las 38 semanas de gestación, juntamente con el uso de terapia antirretroviral materna en el periparto.

El objetivo de la presente investigación fue describir evidencias científicas disponibles de artículos observacionales relacionados a prevalencia y factores asociados de transmisión vertical del VIH durante la gestación.

\section{METODOLOGÍA UTILIZADA}

Tipo de estudio. Investigación tipo revisión de publicaciones de carácter descriptivo.

Población y muestra: Estuvo conformada por 340 artículos originales encontrados en la base de datos electrónicos PubMed, Excerpta Medica Base de datos (EMBASE) y Google Scholar a partir del año 2011 al 2020. La muestra fue constituida por 25 estudios observacionales que cumplieron criterios de inclusión de contar con factores asociados de transmisión vertical durante la gestación.

Técnicas e instrumentos. Se realizó el recojo de la información a partir de la de base de datos electrónicos, para ello se elaboró una ficha de recolección de datos ad hoc, para recoger los datos contemplados en el presente estudio.

Procedimientos. Se usó el formato digital en una base datos en Microsoft Excel versión 2010.

Análisis de datos. Se realizó un análisis descriptivo de los datos resumiendo las variables categóricas de los hallazgos expresados como razón de probabilidad (OR) o riesgos relativos (RR) de cada estudio con sus intervalos de confianza al $95 \%$.

Aspectos éticos. Como los datos recopilados de los estudios científicos ya se encuentran publicadas y validadas, la originalidad y autoría de investigaciones fueron respetadas y debidamente consignadas en las referencias bibliográficas.

\section{HALLAZGOS}

\section{A. PREVALENCIA DE LA INFECCIÓN CON VIH EN GESTANTES}

Según reportes de $\mathrm{ACOG}^{2}$ la transmisión vertical del VIH, en ausencia de terapia y profilaxis materna se produce en un $14-25 \%$ de los casos en países desarrollados. Dicha transmisión vertical se puede producir intraútero (25-40\% de los casos) o intraparto (60-75\% de los casos). La lactancia materna aumenta el riesgo en un $16 \%$ en casos de infección establecida y en un $29 \%$ en casos de primoinfección ${ }^{2,9}$.

a) Características generales de los estudios seleccionados.

En la tabla 1 se describen las características generales de 25 estudios originales, relacionados a las variables: autor, año de publicación, país, objetivo del estudio, diseño de investigación, tamaño de muestra y porcentaje de prevalencia de transmisión vertical del VIH.

b) Prevalencia de la transmisión vertical del VIH. Como se aprecia en la tabla 2 la prevalencia de transmisión materno infantil del VIH varían según distintos autores y áreas geográficas del estudio, tal es así, los autores Yitayew YA et $\mathrm{al}^{23}$ mencionan el 3,8\% en la región de AmharaEtiopía, Moges AN et $a^{28} 5.9 \%$ hasta $17,0 \%$ con Birlie $\mathrm{B}$ et $\mathrm{al}^{34}$ en región Oromia-Etiopía. 
Tabla 1. Características generales de los estudios observacionales incluidos. Porcentaje de transmisión vertical del VIH durante la gestación.

\begin{tabular}{|c|c|c|c|c|}
\hline Autor/Año/País & Objetivo de estudio & $\begin{array}{c}\text { Diseño de } \\
\text { investigación }\end{array}$ & $\begin{array}{c}\text { Tamaño de } \\
\text { muestra } \\
\text { (mujeres } \\
\text { infectadas con } \\
\text { VIH) }\end{array}$ & $\begin{array}{l}\text { Porcentaje de } \\
\text { Transmisión vertical } \\
\text { (IC al } 95 \%)\end{array}$ \\
\hline $\begin{array}{l}\text { Nguyen RN et al / } \\
\text { 2020/ Vietnam }\end{array}$ & $\begin{array}{l}\text { Determinar la evolución de la tasa de } \\
\text { transmisión del VIH en recién nacidos de } \\
2007 \text { a } 2018 \text { e identificar los factores de } \\
\text { riesgo de transmisión del VIH entre los } \\
\text { recién nacidos expuestos al VIH en Vietnam }\end{array}$ & $\begin{array}{l}\text { Estudio cohorte } \\
\text { prospectiva }\end{array}$ & 472 & $\begin{array}{c}8,9 \% \\
\text { (IC del } 95 \% 6,4-12,0)\end{array}$ \\
\hline $\begin{array}{l}\text { Velásquez - Vasquez } \\
\text { C et all } 2020 \text { / Perú }{ }^{3}\end{array}$ & $\begin{array}{l}\text { Conocer las características de los niños con } \\
\text { VIH por TMI nacidos a partir del } 2012 \text { y que } \\
\text { recibieron atención hasta el primer semestre } \\
\text { del } 2018 \text { en los hospitales de Lima y Callao; } \\
\text { así mismo, identificar puntos que pueden } \\
\text { considerarse críticos para la transmisión del } \\
\text { virus al niño. }\end{array}$ & $\begin{array}{l}\text { Estudio } \\
\text { transversal }\end{array}$ & $\begin{array}{l}68 \text { niños con VIH } \\
\text { por TMI }\end{array}$ & $4 \% \mathrm{TMI}$ \\
\hline $\begin{array}{l}\text { Yitayew YA et al / } \\
\text { 2019/ Etiopía }{ }^{23}\end{array}$ & $\begin{array}{l}\text { Evaluar la transmisión materno infantil del } \\
\text { VIH y los factores asociados entre los recién } \\
\text { nacidos expuestos al VIH. }\end{array}$ & $\begin{array}{l}\text { Estudio } \\
\text { transversal }\end{array}$ & 313 & $\begin{array}{l}\% \text { (IC del } 95 \% \\
1.7-5.9)\end{array}$ \\
\hline
\end{tabular}

Huang K-Y et al / 2019/ Taiwán ${ }^{24}$

Do Prado TN et al /
2018 / Brazil ${ }^{25}$

Posadas-Robledo FJ 2018 / México $^{7}$ Obsaa S et al / 2018 /
Etiopía $^{26}$

Describir el tratamiento clínico de las madres infectadas por el VIH y la tasa de transmisión materno infantil en el Hospital Estudio cohorte Universitario Nacional de Taiwán, Taipei, retrospectiva Taiwán, en los años posteriores al inicio del programa.

Estimar la proporción de transmisión vertical del VIH e identificar los factores asociados con la transmisión de madre a hijo en Brasil.

Evaluar si los fetos extraídos por cesárea electiva de madres VIH positivas tienen Estudio menor frecuencia de positividad en una cohorte prueba rápida al nacimiento que los nacidos retrospectiva por vía vaginal.

\section{Estudio cohorte} retrospectiva

39 gestantes a término
Tasa de TMI del $0 \%$.

$14.0 \%$

(IC del $95 \% 11-17 \%)$

Realizar el seguimiento y determinar la tasa de transmisión del VIH entre los bebés nacidos de madres seropositivas como Estudio cohorte principal indicador para comprender el retrospectiva desempeño de un programa nacional de control del VIH en Etiopía.

Evaluar la tasa de parto vaginal y las indicaciones de cesárea en las mujeres Estudio cohorte que viven con el $\mathrm{VIH}$ durante 20 años en un retrospectiva entorno de baja tasa general de cesárea.
212 mujeres con seguimiento constante de carga viral

\section{1 nacimientos por cesárea electiva}

cesárea electiva, mostró un riesgo relativo de 0.07 con (IC del 95\% 0.060.09 )

$80,0 \%$ gestantes mostró cargas virales del VIH $<50$ copias / $\mathrm{ml}$. El $74,5 \%$ de todas las mujeres que viven con el VIH dieron

a luz por vía vaginal

y la tasa de cesáreas electivas y cesáreas de emergencia fue del $12,8 \%$ cada una

Determinar la tasa de transmisión del VIH y

Moges AN et al / 2017 factores asociados entre los recién nacidos Estudio cohorte / Etiopía ${ }^{28}$

expuestos al $\mathrm{VIH}$ en establecimientos de retrospectiva $5.9 \%$ salud de Etiopía.

Scott $\mathrm{R}$ et all $2017 /$ Latinoamérica y El Caribe (Argentina, Bahamas, Brasil,

México, Perú y Jamaica $)^{29}$

Evaluar si existe un aumento de la transmisión materno infantil del $\mathrm{VIH}-1$ asociado con partos a las 40 semanas de Estudio cohorte edad gestacional estimada (EGA) o más en prospectiva mujeres embarazadas con cargas virales de 1000 copias / ml o menos

Analizar los factores de riesgo asociados a transmisión vertical del VIH en el embarazo en las

Ola DJ et al/2017/ Guatemala $^{30}$ pacientes que asisten al Hospital de Estudio

Ginecología y Obstetricia del Instituto transversal

Guatemalteco de Seguridad Social durante

el período 2004 al 2013
305

(IC del 95\% 3.3-8.5)

a) Tasa global de

transmisión $0,4 \%$

(IC del 95\% 0,2-8,1\%)

1,630 gestantes de 40 a 42 semanas

b) A 40 semanas de EG o más $0,5 \%$

(IC del 95\% 0,2-1,4\%);

c) $<40$ semanas EG $0,3 \%$ (IC del 95\% 0,1-0,7\%);

Principal factor que favorece la transmisión vertical la lactancia materna con un Razón de

prevalencia: 6.8

(IC del 95\% 1.17- 39) 
Investigar los cambios en la carga viral de Myer L et al / 2017 / V VIH(LV) materna durante el embarazo y la Sudáfrica ${ }^{31}$ transmisión materno infantil (TMI) tras el inicio de la terapia antirretroviral (TAR) en Ciudad del Cabo, Sudáfrica.
Riesgo de transmisión materno infantil:

$\begin{array}{lcc}\text { Estudio cohorte } & \begin{array}{c}620 \text { mujeres } \\ \text { embarazadas } \\ \text { infectadas por el }\end{array} & \text { a) a) CV }>1000 \text { copias } / \mathrm{ml} \\ \text { prospectiva } & \text { b) CV } 50-1000 \text { copias } / \mathrm{ml} \\ & \text { VIH } & \text { TMI } 2.5 \% \\ & & \text { c) CV }<50 \text { copias } / \mathrm{ml} \mathrm{TMI} \\ & & 0.25 \%\end{array}$

\section{Evaluar la eficacia de los programas de}

Abdula M et al /2017/ prevención de la transmisión materno infantil Estudio Etiopía 32 mediante la determinación del resultado de transversal los recién nacidos expuestos al VIH.

$7.7 \%$ (IC al $95 \% 3.1-12.3)$

La tasa global de TMI para gestantes A Término que dieron a luz con una 2.116 embarazos quración de RPM $\geq 4$ a término en horas fue de $0,64 \%$ en pacientes comparación con $0,34 \%$ tratados con ART para RPM $<4$ horas, sin combinada diferencias significativas entre los grupos (OR 1,90; IC del 95\%: 0,45 a 7,97)

Peters H et al / 2016

Investigar la asociación entre la duración de Irlanda ${ }^{33}$ de transmisión del VIH de madre a hijo (MTCT) en la era de la terapia antirretroviral combinada (CART).
Estudio cohorte prospectiva

\begin{tabular}{|c|c|c|c|c|}
\hline $\begin{array}{l}\text { Birlie B et al / 2016/ } \\
\text { Etiopía }^{34}\end{array}$ & $\begin{array}{l}\text { Cuantificar la tasa de transmisión materno } \\
\text { infantil y sus predictores entre los recién } \\
\text { nacidos expuestos al VIH de gestantes } \\
\text { positivas en un hospital especializado de } \\
\text { Etiopía }\end{array}$ & $\begin{array}{l}\text { Estudio cohorte } \\
\text { retrospectiva }\end{array}$ & 146 & $\begin{array}{c}17.0 \% \text { (IC del } 95 \% \\
10.9-23.1)\end{array}$ \\
\hline $\begin{array}{l}\text { Wudineh F et al / } 2016 \\
\text { / Etiopía }\end{array}$ & $\begin{array}{l}\text { Determinar la transmisión materno infantil } \\
\text { de la infección por VIH y sus determinantes } \\
\text { entre los recién nacidos expuestos al } \\
\text { VIH que reciben atención en Hospital de } \\
\text { Referencia de Etiopía }\end{array}$ & $\begin{array}{l}\text { Estudio cohorte } \\
\text { retrospectiva }\end{array}$ & 382 & $\begin{array}{c}15.7 \% \\
\text { (IC al 95\% 12.1-19.3) }\end{array}$ \\
\hline $\begin{array}{l}\text { Tigabu Z et al / } 2016 \text { / } \\
\text { Etiopía }^{36}\end{array}$ & $\begin{array}{l}\text { Evaluar la proporción de infección por } \\
\text { VIH; niños vinculados a cuidados crónicos } \\
\text { y determinantes de la infección por VIH en } \\
\text { establecimientos de salud de Etiopía. }\end{array}$ & $\begin{array}{l}\text { Estudio } \\
\text { transversal }\end{array}$ & 484 & $\begin{array}{c}12.4 \% \\
\text { (IC al 95\% 1.5-9.5) }\end{array}$ \\
\hline $\begin{array}{l}\text { Negash TG et al } \\
\text { /2016/ Etiopía }{ }^{37}\end{array}$ & $\begin{array}{l}\text { Evaluar los resultados de salud (profilaxis } \\
\text { antirretroviral versus tratamiento } \\
\text { antirretroviral, recuentos de CD4, etapas } \\
\text { de la enfermedad de la OMS, otras } \\
\text { enfermedades) de las mujeres que habían } \\
\text { utilizado estos servicios, así como el estado } \\
\text { del VIH de sus bebés y el método de } \\
\text { alimentación infantil adoptado. }\end{array}$ & $\begin{array}{l}\text { Estudio } \\
\text { transversal }\end{array}$ & 384 & $\begin{array}{c}6.0 \\
\text { (IC al 95\% 3.6-11.3) }\end{array}$ \\
\hline $\begin{array}{l}\text { Girma M et al /2016/ } \\
\text { Etiopía }^{38}\end{array}$ & $\begin{array}{l}\text { Evaluar la aceptación de la intervención de } \\
\text { prevención de la transmisión materno infantil } \\
\text { por parte de las madres embarazadas VIH } \\
\text { positivos y evaluar el grado de vinculación } \\
\text { de los bebés expuestos al VIH. }\end{array}$ & $\begin{array}{l}\text { Estudio cohorte } \\
\text { prospectiva }\end{array}$ & 435 & $\begin{array}{c}0.7 \% \\
\text { (IC al } 95 \%-0.08-1.5)\end{array}$ \\
\hline $\begin{array}{l}\text { Mandelbrot } L \text { et al / } \\
2015 \text { en Francia }{ }^{6}\end{array}$ & $\begin{array}{l}\text { Determinar si el inicio de la terapia } \\
\text { antirretroviral de gran actividad (TAR) antes } \\
\text { de la concepción tiene el potencial de } \\
\text { eliminar la transmisión perinatal (TP) }\end{array}$ & $\begin{array}{l}\text { Estudio cohorte } \\
\text { prospectiva }\end{array}$ & 2651 mujeres & $\begin{array}{c}\text { Tasa global de TP } 0.7 \% \\
\text { No hubo ningún caso de } \\
\text { TP (IC del 95\%: 0,0-0,1) } \\
\text { entre las } 2651 \text { mujeres } \\
\text { que comenzaron el TAR } \\
\text { antes de la concepción, } \\
\text { lo continuaron durante el } \\
\text { embarazo y dieron a luz } \\
\text { con una carga viral <50 } \\
\text { copias / ml }\end{array}$ \\
\hline $\begin{array}{l}\text { Kumela K et al /2015/ } \\
\text { Etiopía }^{39}\end{array}$ & $\begin{array}{l}\text { Determinar la eficacia de los regímenes } \\
\text { antirretrovirales de corta duración y } \\
\text { antirretrovirales de gran actividad en la } \\
\text { prevención de la transmisión materno } \\
\text { infantil del VIH y los factores asociados. }\end{array}$ & $\begin{array}{l}\text { Estudio cohorte } \\
\text { retrospectiva }\end{array}$ & 180 & $\begin{array}{c}15.5 \% \\
\text { (IC al 95\% 10.2-20.8) }\end{array}$ \\
\hline $\begin{array}{l}\text { Townsend C et al I } \\
2014 \text { / Reino Unido e } \\
\text { Irlanda }{ }^{21} \text {. }\end{array}$ & $\begin{array}{l}\text { Analizar las tasas de transmisión del VIH } \\
\text { de madre a hijo (TMH) a lo largo del tiempo } \\
\text { a la luz de los cambios en el manejo, } \\
\text { las características demográficas y del } \\
\text { embarazo, periodos 2000-2001 y 2010-2011 }\end{array}$ & $\begin{array}{l}\text { Estudio cohorte } \\
\text { retrospectiva }\end{array}$ & $\begin{array}{c}12486 \\
\text { embarazos únicos }\end{array}$ & $\begin{array}{l}\text { La tasa de TMI disminuyó } \\
\text { del } 2,1 \% \text { en } 2000-2001 \\
\text { al 0,46\% en } 2010-2011 \\
\text { debido a una combinación } \\
\text { de factores, incluido el } \\
\text { inicio más temprano de } \\
\text { la terapia antirretroviral } \\
\text { combinada prenatal } \\
\text { (TARc) } \\
\end{array}$ \\
\hline
\end{tabular}




\begin{tabular}{|c|c|c|c|c|}
\hline $\begin{array}{l}\text { Briand } \mathrm{N} \text { et al / 2013/ } \\
\text { Francia } 40\end{array}$ & $\begin{array}{l}\text { Describir el modo de parto en mujeres } \\
\text { infectadas VIH y su asociación con la } \\
\text { transmisión materno infantil según carga } \\
\text { viral. }\end{array}$ & $\begin{array}{l}\text { Estudio cohorte } \\
\text { retrospectiva }\end{array}$ & $\begin{array}{l}4300 \\
\text { gestantes a } \\
\text { término }\end{array}$ & $\begin{array}{c}\text { Carga viral }<50 \text { copias } / \mathrm{ml} \text { : } \\
\text { parto vaginal y cesárea } \\
\text { electiva } 0.3 \% \text {. } \\
\text { Carga viral }>10,000 \\
\text { copias/ml: vaginal } 4.0 \% \text { y } \\
\text { cesárea electiva } 5.3 \%\end{array}$ \\
\hline $\begin{array}{l}\text { Briand N et al / 2013/ } \\
\text { Francia } 41\end{array}$ & $\begin{array}{l}\text { Evaluar el impacto de la zidovudina (ZDV) } \\
\text { intravenosa según la carga viral y las } \\
\text { condiciones obstétricas. }\end{array}$ & $\begin{array}{l}\text { Estudio cohorte } \\
\text { retrospectiva }\end{array}$ & $\begin{array}{l}11538 \\
\text { partos }\end{array}$ & $\begin{array}{c}\text { Gestante con carga viral } \\
\geq 1000 \text { copias / ml, la tasa } \\
\text { global de Transmisión } \\
\text { vertical fue } 7.5 \% \text { sin ZDV } \\
\text { intravenosa frente a 2,9\% } \\
\text { con ZDV intravenosa. }\end{array}$ \\
\hline $\begin{array}{l}\text { Velásquez C / 2011/ } \\
\text { Perú }^{18}\end{array}$ & $\begin{array}{l}\text { Evaluar el impacto de la aplicación de tres } \\
\text { guías nacionales para la prevención de la } \\
\text { transmisión vertical del VIH de tres periodos } \\
\text { sucesivos entre los años } 1996 \text { al } 2009 \text { en el } \\
\text { Instituto Nacional Materno Perinatal }\end{array}$ & $\begin{array}{l}\text { Estudio cohorte } \\
\text { retrospectiva }\end{array}$ & $\begin{array}{c}275 \\
\text { nacimientos }\end{array}$ & $15 \%$ disminuyó a $4 \%$ \\
\hline \multicolumn{2}{|c|}{ TOTAL DE INVESTIGACIONES: 25} & TOTAL DE M & STRA: 41,535 & \\
\hline
\end{tabular}

Leyenda: TMI: Transmisión maternoinfantil. CV: Carga viral. EG: Edad gestacional. RPM: Ruptura prematura de membranas. TP: Transmisión perinatal. TAR: Terapia antirretroviral. ZDV: Zidovudina. . IC: Intervalo de confianza.

Recientes publicaciones como de Nguyen RN et $\mathrm{al}^{5}$ reportan una prevalencia de transmisión vertical en $8,9 \%$. Reportes de Myer $L$ et al ${ }^{31}$ en Sudáfrica el riesgo de transmisión materno infantil (TMI) depende de la carga viral (CV): CV >1000 copias/ml TMI 8.5\%; CV 50-1000 copias/ml TMI $2.5 \%$ y CV $<50$ copias/ml TMI fue $0.25 \%$. Reportes en Perú por Velásquez $\mathrm{C}^{18}$ en el Instituto Nacional Materno Perinatal desde 1996 hasta año 2009, se han aplicado tres guías terapéuticas nacionales para la prevención de la transmisión vertical del VIH cuyo hallazgo mostró que la TMI de $15 \%$ disminuyó a 4\%. Asimismo, recientes reportes de Velasquez - Vasquez $\mathrm{C}$ et $\mathrm{a}^{\beta}$ de la información obtenida de la Dirección de Prevención y Control del VIH, ITS y Hepatitis (DPVIH) y del Centro Nacional de Epidemiología Prevención y Control de Enfermedades (CDC MINSA), todavía existe una incidencia de transmisión vertical de aproximadamente $4 \%$.

Tabla 2. Prevalencia de transmisión vertical del VIH de los estudios seleccionados según autor, país y año de publicación.

\begin{tabular}{|c|c|c|}
\hline Autor / País & Año & $\begin{array}{l}\text { Prevalencia (\%) de transmisión de VIH de madre a hijo } \\
\text { (IC al 95\%) }\end{array}$ \\
\hline Velasquez C et al / Perú ${ }^{3}$ & 2020 & $4 \% \mathrm{TMl}$ \\
\hline Nguyen RN et al / Vietnam ${ }^{5}$ & 2020 & $8.9 \%$ (IC del $95 \% 6.4-12.0)$ \\
\hline Yitayew YA et al/Etiopía ${ }^{23}$ & 2019 & $3.8 \%$ (IC del $95 \% 1.7-5.9)$ \\
\hline Do Prado TN et al / Brazil|25 & 2018 & $14.0 \%$ (IC del $95 \% 11-17)$ \\
\hline Obsaa S et all Etiopía ${ }^{26}$ & 2018 & $7.7 \%$ \\
\hline Moges AN et all Etiopía ${ }^{28}$ & 2017 & $5.9 \%$ (IC del $95 \% 3.3-8.5)$ \\
\hline Peters $\mathrm{H}$ et al / Reino Unido e Irlanda ${ }^{33}$ & 2017 & $\begin{array}{l}\text { RPM } \geq 4 \text { horas: } 0.14 \% \text { en comparación RPM }<4 \text { horas: } 0.12 \% \\
\text { (OR } 1,14 \text {; IC del } 95 \% \text { : } 0,07-18,27 \text { ). }\end{array}$ \\
\hline Myer L et al / Sudáfrica ${ }^{31}$ & 2017 & $\begin{array}{l}\text { Riesgo de transmisión materno infantil: } \\
\text { a) CV }>1000 \mathrm{copias} / \mathrm{ml} \text { TMI } 8.5 \% \\
\text { b) CV } 50-1000 \mathrm{copias} / \mathrm{ml} \text { TMI } 2.5 \% \\
\text { c) CV }<50 \text { copias } / \mathrm{ml} \mathrm{TMI} 0.25 \%\end{array}$ \\
\hline Ola DJ et al / Guatemala30 & 2017 & $\begin{array}{l}\text { Lactancia materna favorece la transmisión vertical con Razón } \\
\text { de Prevalencia: } 6.8 \text { (IC del 95\% 1.17-39) }\end{array}$ \\
\hline $\begin{array}{l}\text { Scott } R \text { et al / Latinoamérica y El Caribe (Argentina, } \\
\text { Bahamas, Brasil, México, Perú y Jamaica) }{ }^{29}\end{array}$ & 2017 & $\begin{array}{l}\text { Tasa global de transmisión } 0.4 \% \text { (IC del 95\% 0.2-8.1\%). A } 40 \\
\text { semanas de EG o más } 0.5 \% \text { (IC del 95\% 0.2-1.4\%); }\end{array}$ \\
\hline Birlie B et al / Etiopía ${ }^{34}$ & 2016 & $17.0 \%(\mathrm{IC}$ del $95 \% 10.9-23.1)$ \\
\hline Wudineh F et al / Etiopía ${ }^{35}$ & 2016 & $15.7 \%$ (IC del 95\% $12.1-19.3)$ \\
\hline Tigabu Z et al / Etiopía ${ }^{36}$ & 2016 & $12.4 \%$ (IC del $95 \% 1.5-9.5)$ \\
\hline Negash TG et al / Etiopía ${ }^{37}$ & 2016 & $6.0 \%($ IC del $95 \% 3.6-11.3)$ \\
\hline Girma M et all Etiopía ${ }^{38}$ & 2016 & $0.7 \%($ IC del $95 \%-0.08-1.5)$ \\
\hline Abdula $\mathrm{M}$ et al / Etiopía ${ }^{32}$ & 2015 & $7.7 \%$ (IC del 95\% 3.1-12.3) \\
\hline Kumela K et al / Etiopía ${ }^{39}$ & 2015 & $15.5 \%$ (IC del 95\% 10.2-20.8) \\
\hline Mandelbrot L et al / Francia ${ }^{6}$ & 2015 & Tasa global de TMI: $0.7 \%$ \\
\hline Velasquez C / Perú18 & 2011 & TMI de $15 \%$ disminuyó a $4 \%$ \\
\hline
\end{tabular}

Leyenda: TMI: Transmisión maternoinfantil. CV: Carga viral. EG: Edad gestacional. RPM: Ruptura prematura de membranas. IC: Intervalo de confianza. 


\section{FACTORES DE RIESGO ASOCIADOS A LA TRANSMISIÓN VERTICAL DEL VIH EN GESTANTES INFECTADAS}

a) Ausencia de terapia antirretroviral durante la atención prenatal en gestante con VIH

Como se aprecia en la tabla 3 la ausencia de terapia con antirretrovirales a la gestante infectada con VIH o no recibieron terapia antirretroviral durante la atención prenatal demuestran que existe asociación de incremento de riesgo de transmisión vertical del VIH según distintos autores tales como reportan Yitayew YA et $\mathrm{al}^{23}$, Do Prado TN et $\mathrm{al}^{25}$, Obsaa S et $\mathrm{al}^{26}$, Abdula $M$ et al ${ }^{32}$ y Wudineh $F$ et al ${ }^{35}$ quienes encontraron razón de probabilidad ORa de 5.70, 4.19, 2.41,17.20 y 4.42 respectivamente. En el Perú, reciente reporte de Velasquez-Vasquez $C$ et $\mathrm{al}^{3}$ de los 68 niños con $\mathrm{VIH}$ por TMI, la madre de 57 de ellos $(83,8 \%)$ no había recibido tratamiento antirretroviral durante el embarazo.

Tabla 3. Ausencia de terapia antirretroviral durante la atención prenatal como factor de riesgo de transmisión vertical según distintos estudios seleccionados.

\begin{tabular}{|c|c|}
\hline Autor/año & $\begin{array}{c}\text { RESULTADOS de TMI } \\
\text { Razón de probabilidad ajustada } \\
\text { (ORa) con Intervalo de Confianza } \\
\text { del } 95 \%\end{array}$ \\
\hline $\begin{array}{l}\text { Velasquez-Vasquez C } \\
\text { et al / } 2020^{3}\end{array}$ & $\begin{array}{l}\text { De los } 68 \text { niños con VIH por TMI, } \\
\text { la madre de } 57 \text { de ellos }(83,8 \%) \\
\text { no había recibido tratamiento } \\
\text { antirretroviral durante el embarazo }\end{array}$ \\
\hline Yitayew YA et al / $2019^{23}$ & 5.70 (IC del 95\% 1.10-29.36) \\
\hline $\begin{array}{l}\text { Do Prado TN et al / } \\
2018^{25}\end{array}$ & 2.41 (IC del 95\% 1,09-5,31) \\
\hline Obsaa S et al /2018 ${ }^{26}$ & 4.19 (IC del 95\% $1.40-12.57)$ \\
\hline Abdula M et al / $2017^{32}$ & 17.20 (IC del $95 \%-31.55-65.95)$ \\
\hline Wudineh F et al / $2016^{35}$ & 4.42 (IC del 95\% 0.52-8.32) \\
\hline
\end{tabular}

b) Ausencia de programa de prevención con antirretrovirales maternos antes o durante el embarazo y ausencia de control prenatal en gestante con VIH.

Como se aprecia en la tabla 4 la ausencia de programa de prevención con uso de antirretrovirales maternos antes o durante el embarazo en la gestante infectada con VIH demuestran que existe asociación de incremento de riesgo de transmisión vertical del VIH según distintos autores tales como reportan Nguyen $\mathrm{RN}$ et $\mathrm{al}^{5}$, Moges $\mathrm{AN}$ et al ${ }^{28}$ y Birlie $B$ et $a^{34}$ quienes encontraron una razón de probabilidad ajustada ORa de 40.6, 15.95 y 4.90 respectivamente, de presentar transmisión vertical del VIH cuando no existe programa de prevención con antirretrovirales en comparación con otros lugares donde si cuentan con dicho programa. Asimismo, según Yitayew $\mathrm{YA}$ et $\mathrm{al}^{23}$ muestra $\mathrm{ORa}=4,6$ cuando la gestante no acude a su atención prenatal en comparación con aquellas gestantes que sí acuden a su control prenatal.

Tabla 4. Ausencia de programa de prevención con antirretrovirales maternos antes o durante el embarazo y ausencia de control prenatal como factor de riesgo de transmisión vertical según estudios seleccionados

\begin{tabular}{cc}
\hline \multicolumn{1}{c}{ Autor/año } & $\begin{array}{c}\text { RESULTADOS de TMI } \\
\text { Razón de probabilidad } \\
\text { ajustada (ORa) con IC del 95\% }\end{array}$ \\
\hline $\begin{array}{c}\text { Ausencia de programa de prevención con antirretrovirales } \\
\text { maternos antes o durante el embarazo }\end{array}$ \\
\hline $\begin{array}{c}\text { Nguyen RN et al / } 2020^{5} \\
\text { Moges AN et al } 2017^{28}\end{array}$ & 40.6 (IC del 95\% 5.5 - 308) \\
\hline Birlie B et al 2016 ${ }^{34}$ & 4.95 (IC del 95\% 3.35 -75.0) \\
\hline Ausencia de visita de atención prenatal materna \\
\hline Yitayew YA et al /201923 & 4,6 (IC del 95\%: 1.17 - 17.99) \\
\hline
\end{tabular}

c) Ausencia de profilaxis antirretroviral al recién nacido de madre infectada por VIH

Como se aprecia en la tabla 5 la ausencia de profilaxis antirretroviral al recién nacido de madre infectada por VIH demuestra que existe asociación de incremento de riesgo de transmisión vertical del VIH según distintos autores tales como reportan Nguyen RN et $\mathrm{al}^{5}$, Wudineh $\mathrm{F}$ et $\mathrm{al}^{35}$, Yitayew YA et $\mathrm{al}^{23}$ y Obsaa S et $\mathrm{al}^{26}$ quienes encontraron un ORa de $3.4 ; 5.83 ; 5.3$ y 18.1 respectivamente. Asimismo, según reporte de Velasquez-Vasquez $\mathrm{C}$ et $\mathrm{al}^{3}$ de 68 niños que recibieron terapia antirretroviral (TARV), 5 de ellos tuvieron falta del TARV desde inicio (7.4\%).

Tabla 5. Ausencia de profilaxis antirretroviral al recién nacido de madre infectada por VIH como factor de riesgo de transmisión según estudios seleccionados

\begin{tabular}{|c|c|}
\hline Autor/año & $\begin{array}{l}\text { RESULTADOS de TMI } \\
\text { Razón de probabilidad ajustada } \\
\text { (ORa) con IC del } 95 \%\end{array}$ \\
\hline Nguyen RN, et al / $2020^{5}$ & 3,4 (IC del 95\% 1,1-10,3) \\
\hline Yitayew YA et al /2019²3 & $\begin{array}{c}5,3 \text { (IC del } 95 \%: 1,11-25,44) \\
\text { imposibilidad de iniciar la profilaxis } \\
\text { con nevirapina en el lactante }\end{array}$ \\
\hline Obsaa S et al /2018 & $\begin{array}{c}18,110 \text { (IC al } 95 \% 5,77-63,352) \\
\text { Fracaso infantil para recibir } \\
\text { profilaxis con nevirapina }\end{array}$ \\
\hline Wudineh F, et al / $2016^{35}$ & 5,83 (IC al 95\% 1,28 -10,38) \\
\hline Velasquez C et al / $2020^{3}$ & $\begin{array}{l}\text { De } 68 \text { niños que recibieron terapia } \\
\text { antirretroviral, } 5 \text { de ellos tuvieron } \\
\text { falta del TARV de inicio }(7,4 \%)\end{array}$ \\
\hline
\end{tabular}


d) Parto domiciliario y cesárea de emergencia en gestante con VIH

Como se aprecia en la tabla 6 en relación al parto domiciliario de gestante infectada por $\mathrm{VIH}$ demuestran que existe asociación de incremento de riesgo de transmisión vertical del $\mathrm{VIH}$ según distintos autores tales como reportan Yitayew YA et $a^{23}$, Wudineh $\mathrm{F}$ et $\mathrm{al}^{35}$, Abdula $\mathrm{M}$ et $\mathrm{al}^{32}$ con ORa 8.0 y Birlie $\mathrm{B}$ et $\mathrm{al}^{34}$ quienes encontraron razón de probabilidad ajustada (ORa) de $4.20,3.35$ y 8.10 respectivamente, en aquellas gestantes que dieron luz en su domicilio en comparación con parto institucional. Asimismo, en relación con la cesárea de emergencia en gestante infectada con VIH se muestra que existe asociación de incremento de riesgo de transmisión vertical del $\mathrm{VIH}$ según el autor Do Prado TN et $a^{25}$ quien reporta un ORa de 4.32.

Tabla 6. Parto domiciliario y cesárea de emergencia como factor de riesgo asociado con la transmisión vertical del $\mathrm{VIH}$

\begin{tabular}{|c|c|}
\hline Autor/año & $\begin{array}{c}\text { RESULTADOS de TMI } \\
\text { Razón de probabilidad ajustada } \\
\text { (ORa) con IC al } 95 \%\end{array}$ \\
\hline \multicolumn{2}{|c|}{ PARTO DOMICILIARIO } \\
\hline Yitayew YA et al / $2019^{23}$ & 4.20 (IC al $95 \%-3.66-12.06)$ \\
\hline Wudineh F et al / $2016^{35}$ & 3.35 (IC al $95 \%-0.05-6.75)$ \\
\hline Birlie B et al / $2016^{34}$ & 8.10 (IC al 95\% 2.10-31.9) \\
\hline Abdula M et al / $2015^{32}$ & 8.0 \\
\hline \multicolumn{2}{|c|}{ CESÁREA DE EMERGENCIA } \\
\hline Do Prado TN et al $/ 2020^{25}$ & $1.32 \mathrm{IC}$ al $95 \% 1.57-11.9)$ \\
\hline
\end{tabular}

e) Carga viral y modo de parto como factor de riesgo de transmisión vertical del VIH

Como se aprecia en la tabla 7 , la carga viral en gestantes con $\mathrm{VIH}$ que se encuentran con terapia antirretroviral presenta distintos valores en riesgo de transmisión materno infantil del VIH según los autores como Briand $\mathrm{N}$ et $\mathrm{a} \mathrm{A}^{40}, 5.3 \%$ en cesárea electiva y Briand $\mathrm{N}$ et $a^{41} 4.7 \%$ en parto vaginal, con carga viral $>10.000$ copias $/ \mathrm{ml}$; asimismo, alcanza $7.5 \%$ de TMI sin zidovudina intravenosa durante el parto o cesárea con carga viral $>1000$ copias/ml. Mientras, Townsend C et al en Reino Unido e Irlanda ${ }^{21}$ en 12486 embarazos únicos con carga viral (CV) de 50-399 copias $/ \mathrm{ml}$, el riesgo de TMl fue del $0,26 \%$ después de una cesárea electiva y del $1,1 \%$ después del parto vaginal planificado. Por otro lado, Myer $L$ et $a l^{31}$ arroja $8.5 \%$ de TMl con carga viral $>1000$ copias $/ \mathrm{ml}$. Sin embargo, con carga viral $<50$ copias/ml según Myer et $\mathrm{al}^{31}$, arroja solo $0.25 \%$ de riesgo de $\mathrm{TMl}$ e incluso según Aho $\mathrm{I}$ et $\mathrm{al}^{27}$, no hay transmisión vertical con carga viral indetectable.

f) Lactancia materna y mixta como factor de riesgo de la transmisión madre-hijo del VIH

Como se aprecia en la tabla 8 en relación con lactancia materna y mixta como factor de riesgo de la transmisión madre-hijo del VIH demuestran que existe asociación de incremento de riesgo de transmisión vertical del VIH según distintos autores tales como reportan Obsaa $S$ et $\mathrm{al}^{26}$, Birlie $B$ et $\mathrm{al}^{34} \mathrm{y}$ Wudineh $\mathrm{F}$ et $\mathrm{al}^{35} \mathrm{y}$ quienes encontraron un ORa de 2.3, $5.60 \mathrm{y}$ 42.21 respectivamente. Asimismo, según Ola DJ et al ${ }^{30}$ lactancia materna favorece la transmisión vertical con razón de prevalencia de 6.8 .

Tabla 7. Carga viral y modo de parto como factor de riesgo de transmisión vertical del VIH

\begin{tabular}{|c|c|c|c|}
\hline Autor/año/país & $\begin{array}{c}\text { Muestra } \\
\text { (Gestantes con VIH) }\end{array}$ & $\begin{array}{l}\text { Carga viral } \\
\text { (copias } / \mathrm{ml} \text { ) }\end{array}$ & $\begin{array}{c}\text { Riesgo de Transmisión vertical según el tipo de } \\
\text { parto }\end{array}$ \\
\hline $\begin{array}{l}\text { Briand } \mathrm{N} \text { et al / 2013/ } \\
\quad \text { Francia4 }{ }^{2} \text { ) }\end{array}$ & $\begin{array}{c}4300 \text { gestantes a } \\
\text { término }\end{array}$ & $\geq 10.000$ copias/ml. & $\begin{array}{l}5.3 \% \text { en cesárea electiva frente a } 4.0 \% \text { en parto } \\
\text { vaginal }\end{array}$ \\
\hline $\begin{array}{l}\text { Briand N et al / 2013/ } \\
\qquad \text { Francia41 }\end{array}$ & 11538 partos & $\begin{array}{c}\text { Gestante con carga viral } \geq \\
1000 \text { copias / ml durante el } \\
\text { parto }\end{array}$ & $\begin{array}{l}\text { La tasa global de Transmisión vertical fue } 7.5 \% \text { sin } \\
\text { ZDV intravenosa frente a } 2,9 \% \text { con ZDV intravenosa, } \\
\text { durante el parto vaginal o cesárea. }\end{array}$ \\
\hline $\begin{array}{l}\text { Townsend C et al / } 2014 \text { / } \\
\text { Reino Unido e Irlanda21 }\end{array}$ & $\begin{array}{l}12486 \text { embarazos } \\
\text { únicos }\end{array}$ & $\begin{array}{l}\text { Carga viral (CV): } \\
\text { a) de } 50-399 \text { copias } / \mathrm{ml} \text { en } \\
\text { cesárea electiva y parto } \\
\text { vaginal }\end{array}$ & $\begin{array}{l}\text { a) El riesgo de TMI fue del } 0,26 \% \text { después de una } \\
\text { cesárea electiva y del } 1,1 \% \text { después del parto vag- } \\
\text { inal planificado. }\end{array}$ \\
\hline $\begin{array}{l}\text { Myer L et al / } 2017 \text { / } \\
\text { Sudáfrica }\end{array}$ & $\begin{array}{c}620 \text { mujeres } \\
\text { embarazadas } \\
\text { infectadas por el VIH }\end{array}$ & $\begin{array}{l}\text { Carga viral (CV) al momento } \\
\text { del parto: } \\
\text { b) }>1000 \text { copias } / \mathrm{ml} \\
\text { c) } 50-1000 \text { copias } / \mathrm{ml} \\
\text { d) }<50 \text { copias } / \mathrm{ml}\end{array}$ & $\begin{array}{l}\text { Riesgo de transmisión materno infantil: } \\
\text { a) } 8.5 \% \\
\text { b) } 2.5 \% \\
\text { c) } 0.25 \% \\
\text { El riesgo de TMI temprana estuvo fuertemente aso- } \\
\text { ciado con la carga viral del parto }\end{array}$ \\
\hline $\begin{array}{l}\text { Aho I et all } 2018 \text { / } \\
\text { Finlandia } 27\end{array}$ & 212 mujeres & $\begin{array}{l}\text { Carga viral del } \mathrm{VIH}<50 \text { copias } \\
\text { / ml en la última medición } \\
\text { anterior al parto. }\end{array}$ & $\begin{array}{l}\text { No se produjeron transmisiones perinatales del VIH } \\
\text { durante el parto vaginal o cesárea }\end{array}$ \\
\hline
\end{tabular}


Tabla 8. Lactancia materna y mixta como factor de riesgo de la transmisión madre-hijo del VIH

\begin{tabular}{lc}
\hline \multicolumn{1}{c}{ Autorlaño } & $\begin{array}{c}\text { RESULTADOS de TMI } \\
\text { Razón de probabilidad ajustada } \\
\text { (ORa) con IC al 95\% }\end{array}$ \\
\hline Obsaa S et al /201826 & 2.3 (IC al 95\% 1.167 - 4.539) \\
Wudineh F et al / $2016^{35}$ & 42.21 (IC al 95\% -60.82 - 145.24) \\
Birlie B et al /201634 & 5.60 (IC al 95\% 1.4 - 41.2) \\
Ola DJ et al/201730 & $\begin{array}{c}\text { Lactancia materna favorece la } \\
\text { transmisión vertical con Razón de } \\
\text { Prevalencia: 6.8 (IC del 95\% 1.17-39) }\end{array}$ \\
\hline
\end{tabular}

\section{CONCLUSIONES}

La prevalencia de transmisión vertical del VIH oscila entre $3,8 \%$ a $17,0 \%$ según los estudios seleccionados. En el Perú, se reporta $4 \%$.

La ausencia del programa de prevención de transmisión de madre a hijo del VIH y ausencia de terapia antirretroviral durante la atención prenatal están asociadas para transmisión vertical del VIH con razón de posibilidad ajustada ORa que varían entre 4,90 a 40,6 y 2,41 a 17,20 respectivamente, según los estudios seleccionados. En el Perú, de 68 niños con VIH por TMI, la madre de 57 de ellos $(83,8 \%)$ no había recibido tratamiento durante el embarazo

El parto domiciliario y cesárea de emergencia presentan razón de posibilidad ajustada ORa que varían entre 3,35 a 8,10 y 4,32 respectivamente, según los estudios seleccionados.

La ausencia de profilaxis antirretroviral al recién nacido de madre infectada por VIH está asociada a la transmisión vertical del VIH con razón de posibilidad ajustada ORa que varían entre 3,4 a 5,83 según los estudios seleccionados.

Financiamiento: Autofinanciado.

Declaración de conflictos de intereses: Los autores declaran no tener conflicto de intereses.

\section{REFERENCIAS BIBLIOGRÁFICAS}

1. Organización Mundial de la Salud. Programa de VIH/Sida. Transmisión del VIH de la madre al niño. [Internet] 2020. [citado 16 setiembre 2020]; Disponible en: https://www.who. int/hiv/ topics/mtct/en/. Accessed September 16, 2020.

2. American College of Obstetricians and Gynecologists. Labor and delivery management of women with human immunodeficiency virus infection. ACOG Committee Opinion No. 751. Obstet Gynecol 2018; 2018:131-7.

3. Velásquez-Vásquez C, Espinola-Sánchez M. Caracterización de niños con VIH por transmisión materno-infantil atendidos en hospitales de Lima, Perú. Rev Peru Med Exp Salud
Publica. 2020;37(4):694-99. doi: https:// doi.org/10.17843/ rpmesp.2020.374.4816

4. Organización Mundial de la Salud \& Organización Panamericana de la Salud. Estrategia y plan de acción para la eliminación de la transmisión materno-infantil del VIH y de la sífilis congénita: Estrategia de monitoreo regional. Tercera edición. 2014 [Internet]. Washington DC: OPS; 2014 [citado el 24 de mayo de 2019]. Disponible en: https:// iris.paho. org/bitstream/handle/10665.2/31365/9789275318126-spa. pdf? sequence $=1 \&$ isAllowed $=y$

5. Nguyen RN, Ton QC, Tran QH, Nguyen TKL. Mother-to-Child Transmission of HIV and Its Predictors Among HIV-Exposed Infants at an Outpatient Clinic for HIVIAIDS in Vietnam. HIVIAIDS - Research and Palliative Care. 2020; 12:253261. doi: $10.2147 /$ hiv.s259592

6. Mandelbrot L, Tubiana R, Le Chenadec J, Dollfus C, Faye A, Pannier E, Matheron S, Khuong MA, Garrait V, Reliquet V, Devidas A, Berrebi A, Allisy C, Elleau C, Arvieux C, Rouzioux C, Warszawski J, Blanche S. No Perinatal HIV-1 Transmission From Women With Effective Antiretroviral Therapy Starting Before Conception. Clinical Infectious Diseases,2015: civ578. doi:10.1093/cid/civ578

7. Posadas-Robledo FJ. Embarazo y VIH ¿indicación absoluta de cesárea? Ginecol Obstet Mex.2018; 86(6):374-382. Doi: https://doi.org/10.24245/gom.v86i6.2018

8. Ministerio de Salud. Resolución Ministerial 1138-2019/ MINSA. Norma técnica de salud para la prevención de la transmisión materno infantil del $\mathrm{VIH}$, sífilis y hepatitis. NT N¹59-MINSA-2019/DGIESP/. 2019.

9. Hospital Universitari Clínic Barcelona. Infección por VIH y gestación. Protocolos 2017.

10. López-Cervantes M, Castro-Borbonio MV, Pérez-Enríquez O, Rojas-Russell M. Causales críticas de la trasmisión vertical del VIH y sífilis congénita en México. Una perspectiva basada en estudios. Ciudad de México, 2014. www.censida. salud. gob.mx/descargas/biblioteca/CausalesTVVIHySC.pdf

11. Díaz-Vega A, González-Santes M, Domínguez-Alfonso A, Arias-Contreras A. Factores de riesgo asociados a la transmisión madre-hijo del VIH en los pacientes del CAPACITS de Veracruz. Perinatol Reprod Hum. 2013; 27 (4): 229-234.

12. Portocarrero J. VIH en gestantes indígenas: un reto para la salud pública peruana. Rev Peru Med Exp Salud Publica. 2015;32(3):546-54

13. Perú, Ministerio de Salud; Fondo de Población de las Naciones Unidas. Una aproximación cualitativa a la prevención del VIHSida en dos comunidades nativas de Ucayali. Lima: Instituto de Educación y Salud; 2009.

14. Zavaleta $C$, Fernández $C$, Konda K, Valderrama Y, Vermund $\mathrm{SH}$, Gotuzzo E. High prevalence of HIV and syphilis in a remote native community of the Peruvian Amazon. Am J Trop Med Hyg. 2007;76(4):703-5.

15. Perú, Ministerio de Salud; Dirección General de Epidemiología. Análisis de la situación epidemiológica del VIH/ SIDA en el Perú - 2013. Lima: JB GRAFIC; 2013.

16. Alarcón J, Romaní F, Montano S, Zunt JR. Transmisión vertical de HTLV-1 en el Perú. Rev Peru Med Exp Salud Publica. 2011;28(1):101-8.

17. García PJ, Nureña CR, Bayer AM, Cárcamo C, Lazo $\mathrm{M}$, La Rosa $\mathrm{S}$, et al. Nunca pensé que me iba a pasar a mí. VIH y vulnerabilidad de la mujer en el Perú: evidencias y recomendaciones para la acción [Internet]. 
Lima: Universidad Peruano Cayetano Heredia; 2013 [citado el 28 de mayo de 2019]. Disponible en: http:// www2.congreso.gob.pe/sicr/cendocbib/ con4 uibd.nsf/ C531EFAF98CB3BDF05257BBB005CB3D3/\$FILE/ UPCHONUSIDA-VIH-y-Vulnerabilidad-Mujer-Peru.pdf.

18. Velásquez C. Resultados de la aplicación de tres guías nacionales para prevenir la transmisión vertical del VIH en el Instituto Nacional Materno Perinatal. Lima, Perú. Rev Peru Med Exp Salud Publica. 2011;28(3):492- 496. doi: 10.17843/ rpmesp.2011.283.528.

19. Huamán B, Gushiken A, Benites C, Quiroz F, GarciaFernández L. Prevención de la transmisión materno-infantil del VIH en gestantes y madres awajún y wampis de la región Amazonas en Perú. Rev Peru Med Exp Salud Pública. 2017;34(4):627-32. doi: 10.17843/rpmesp.2017.344.2725.

20. Grández-Urbina JA, Fernández-Luque JL. Etnias de la Amazonía en riesgo de enfermedades de transmisión sexual, una realidad en nuestro país. Rev Med Hered. 2012;23(1):70-6.

21. Townsend CL, Byrne L, Cortina-Borja M, et al. Earlier initiation of ART and further decline in mother-to-child HIV transmisión rates, 2000-2011. AIDS. 2014;28: 1049-57. https://journals.Iww.com/aidsonline/pages/articleviewer. aspx year $=2014 \&$ issue $=04240 \&$ article $=00014 \&$ type $=$ Abstract

22. Expert Panel Secretariat of the National AIDS Plan (PNS); Spanish Society of Gynecology and Obstetrics (SEGO); AIDS Study Group (GeSIDA); Spanish Society of Pediatric Infectious Diseases (SEIP), Polo Rodríguez R, Muñoz Galligo $\mathrm{E}$, et al. Executive summary of the Consensus Statement on monitoring HIV: pregnancy, birth, and prevention of mother-to-child transmission. Enferm Infecc Microbiol Clin. 2014;32(5):311-9. doi: 10.1016/j.eimc.2013.12.006.

23. Yitayew YA, Bekele DM, Demissie BW, Aragew Z. (2019). Mother to child transmission of HIV and associated factors among HIV exposed infants at public health facilities, Dessie Town, Ethiopia. HIVIAIDS - Research and Palliative Care, 2019;11:343-350. doi:10.2147/hiv.s221409.

24. Huang K-H, Li Y-P, Shih Ch-Ch, Lin CH, Kang J, Lin MW, Hsu WW,Tai Y-Y, Lin S-H, Ho H-N. Mother-to-child transmission of HIV: An 11-year experience in a single center and HIV prevention effectiveness in Taiwan. Journal of the Formosan Medical Association. 2019; 118: 1211e1217.

25. Do Prado TN, Brickley DB, Hills NK, Zandonade E, MoreiraSilva SF, Miranda AE. Factors associated with maternal-child transmisión of HIV-1 in Southeastern Brazil: a retrospective study. AIDS Behav. 2018; 22(Suppl 1):92-98. doi:10.1007/ s10461-018-2172-8.

26. Obsaa S, Dabsub R, Ejetab E. Rate of mother to child transmission of HIV and factors associated among HIV exposed infants in Oromia Regional State, Ethiopia: Retrospective study . Egyptian Pediatric Association Gazette. 2018;66: 61-65.

27. Aho I, Kaijomaa M, Kivelä $P$, Surcel H-M, Sutinen J, Heikinheimo O, et al. Most women living with HIV can deliver vaginally —National data from Finland 1993-2013. PLoS ONE.2018;13(3): e0194370. https://doi.org/10.1371/journal. pone. 0194370

28. Moges NA, Kassa GM, Boneya DJ. Rate of HIV transmission and associated factors among HIV-exposed infants in selected health facilities of east and west Gojjam zones, Northwest Ethiopia; retrospective cohort study. BMC Infect Dis. 2017;17(1):475

29. Scott R, Chakhtoura N, Burke M, Cohen R, Kreitchmann R. Delivery After 40 Weeks of Gestation in Pregnant
Women With Well-Controlled Human Immunodeficiency Virus. Obstet Gynecol. 2017;130(3):502-510. doi: 10.1097/ AOG.0000000000002186

30. Ola DJ, Herrarte ER. Factores de riesgo asociados a transmisión vertical del VIH en el embarazo. REVCOG. 2017; 22:(2):38-40

31. Myer L, Phillips TK, McIntyre JA, Hsiao N-Y, Petro G, Zerbe A, Ramjith J, Bekker L-G, Abrams EJ. HIV viraemia and mother-to-child transmission risk after antiretroviral therapy initiation in pregnancy in Cape Town, South Africa. HIV Med. 2017;18(2):80-88. doi: 10.1111/hiv.12397.

32. Abdula M, Tilahun Z, Workneh S. Assessment of effectiveness of prevention of mother to child transmission of human immunodeficiency virus in Asella hospital, Ethiopia. Eur J Clin Pharm. 2017;19(3):198-206.

33. Peters H, Byrne L, De Ruiter A, Francis K, Harding K, Taylor $\mathrm{GP}$, et al. Duration of ruptured membranes and mother-tochild HIV transmission: a prospective population-based surveillance study. BJOG 2016; 123:975-81.

34. Birlie B, Diriba T, Sisay K, Gurmessa A, Seyoum D. Mother to child HIV transmission and its predictors among HIV-exposed infants: a retrospective follow-up study in Southwest Ethiopia. J AIDS Clin Res. 2016;7(605):2.

35. Wudineh F, Damtew B. Mother-to-child transmission of HIV infection and its determinants among exposed infants on care and follow-up in Dire Dawa City, eastern Ethiopia. AIDS Res Treatment 2016; 2016:1-6.

36. Tigabu Z, Wasie B. Outcomes and linkage to chronic care of HIV exposed infants among health centers and hospitals in Amhara region, Ethiopia: implications to prevention of motherto child transmission of HIV program: a cross sectional study. Pan Afr Med J. 2016;24(1).

37. Negash TG, Ehlers VJ. An assessment of the outcomes of prevention of mother-to-child transmission of HIV services in Addis Ababa. Ethiopia Curationis. 2016;39(1):1-9.

38. Girma M. Effectiveness of prevention of mother-to-child transmisión (PMTCT) procedures in pregnant HIV infected women and their exposed infants at seven health centers in Addis Ababa, Ethiopia; University of Munich (LMU); 2016.

39. Kumela K, Amenu D, Chelkeba L. Comparison of anti-retroviral therapy treatment strategies in prevention of mother-to-child transmission in a teaching hospital in Ethiopia. Pharm Pract. $2015 ; 13(2)$

40. Briand N, Jasseron C, Sibiude J, et al. Cesarean section for HIV-infected women in the combination antiretroviral therapies era, 2000-2010. Am J Obstet Gynecol 2013; 209: 335.e1-12. https://www.ncbi.nlm.nih.gov/pubmed/23791563.

41. Briand N, Warszawski J, Mandelbrot L, Dollfus C, Pannier E, Cravello $\mathrm{L}$, et al. Is intrapartum intravenous zidovudine for prevention of mother-to-child HIV-1 transmission still useful in the combination antiretroviral therapy era? ANRS-EPF CO1CO11 Study Group. Clin Infect Dis.2013:57:903-14.

\section{Correspondencia:}

Katherin Faviola Moreno Reyes

Dirección: San Diego Mz. C Lote 18 Valle Azul II etapa-San

Martín de Porres. Lima-31.

E-mail: katherin r28@hotmail.com.

Teléfono: 962198849 